\title{
A Retrospective Study of the Association of Repeated Attempts at Trial of Labor After Cesarean Birth on Maternal and Neonatal Outcomes
}

\author{
Abdulrahim A Rouzi (1) \\ Rana Alamoudi \\ Sarah Ghazali \\ Nisma Almansouri \\ Abdullah Kafy \\ Meshari Alrumaihi \\ Wajeh Hariri \\ Esraa Alsafri
}

Department of Obstetrics and Gynecology, King Abdulaziz University, Jeddah, Saudi Arabia
Correspondence: Abdulrahim A Rouzi PO Box 80215, Jeddah, 21589, Saudi Arabia

Tel +966505602587

Email aarouzi@gmail.com
Purpose: To assess the maternal and neonatal outcomes of repeated trials of labor after one previous cesarean section.

Materials and Methods: We identified and reviewed the records of all women who had had a trial of labor after cesarean section at a tertiary care center in Saudi Arabia between January 1, 2011, and December 30, 2018. The inclusion criteria were women with singleton vertex pregnancies between 24 and 42 weeks of gestation and a trial of labor after one cesarean section. The exclusion criteria were two or more previous cesarean sections, intrauterine fetal demise, breech presentation, labor induction, estimated fetal weight $>4 \mathrm{~kg}$, and classical or low vertical uterine incision. The pregnancy outcomes of these women were compared according to the number of trials of labor after cesarean section.

Results: During the study period, 1139 women met the inclusion criteria. The number of women with previous zero, one, two, or three or more trials of labor after cesarean section were 669 (58.7\%), 237 (20.8\%), 132 (11.6\%), and 101 (8.9\%), respectively. There were statistically significant trends between the four groups in age, nationality, gravidity, and parity but not in the booking status, BMI, or the hemoglobin level before a trial of labor after cesarean section. The rate of vaginal birth after cesarean section increased significantly ( $p<0.001$ ) from $72.9 \%$ with zero to $93.3 \%$ with one, $93.9 \%$ with two, and $94.1 \%$ with three or more trials of labor after cesarean section.

Conclusion: Previously successful vaginal births after cesarean delivery are associated with improved maternal and neonatal outcomes in the subsequent trials of labor after cesarean delivery. Keywords: trial of labor after cesarean, cesarean section

\section{Introduction}

The rate of cesarean delivery has progressively increased across the world. Between 1990 and 2014, the rate of cesarean delivery increased from $22.8 \%$ to $42.2 \%$ in Latin America and the Caribbean, $4.4 \%$ to $19.5 \%$ in Asia, $18.5 \%$ to $32.6 \%$ in Oceania, $11.2 \%$ to $25 \%$ in Europe, $22.3 \%$ to $32.3 \%$ in North America, and $2.9 \%$ to $7.4 \%$ in Africa. ${ }^{1}$ The probable reasons behind this rise include medical (increased maternal age, obesity, breech presentation, failed induction of labor, and a history of prior cesarean section) and non-medical indications (preference of the mother or family for cesarean delivery, clinician convenience, financial incentives, and concerns of medico-legal issues). ${ }^{2-5}$

One of the obstetric sequelae after cesarean section is the high probability of repeat cesarean sections. Several attempts have been made to reduce this 
complication, including a trial of labor after cesarean section. It is an appropriate option for many women as recommended by the American College of Obstetricians and Gynecologists. ${ }^{6}$ They stated that

most women with one previous cesarean delivery with a low-transverse incision are candidates for and should be counseled about and offered a trial of labor after cesarean section.

Similar conclusions were published by the Society of Obstetricians and Gynecologists of Canada and the Royal College of Obstetricians and Gynecologists. ${ }^{7,8}$ A successful trial of labor after cesarean section provides an opportunity to achieve a vaginal birth after cesarean delivery.

In contrast, a failed trial results in an emergency cesarean section with increased maternal morbidity. The rate of maternal morbidity is the lowest in women who have a successful vaginal birth after cesarean delivery $(0.2 \%)$, higher in women who undergo an elective repeat cesarean section $(0.8 \%)$, and highest in women who have a failed trial of labor after cesarean section $(3.3 \%))^{9}$ The advantages of vaginal birth after cesarean include safety, elimination of major surgery, acceptability, and shorter recovery time than with the elective cesarean section. ${ }^{6}$ In women who desire future pregnancies, successful vaginal birth after cesarean avoids the need for multiple cesarean sections with the known risks of surgery, placenta previa, and placenta accreta spectrum.

While the safety of a trial of labor after one previous cesarean section is well-documented, there is a lack of recently published studies on repeat trials of labor after cesarean section. The present study aimed to assess the maternal and neonatal outcomes of repeat trials of labor after one previous cesarean section.

\section{Materials and Methods}

This retrospective study was conducted at a tertiary care center in Saudi Arabia. The hospital records of all women who were admitted to our hospital between January 1, 2011, and December 30, 2018, who had had a trial of labor after cesarean section were identified and reviewed. The study was approved by the King Abdulaziz University Hospital Institutional Review Board (Number 381-17) and performed in accordance with relevant guidelines and regulations in Saudi Arabia and the Declaration of Helsinki. Because of the type of the study, patient consent to review their medical records was not required by the King Abdulaziz University Hospital IRB.
Saudi and all eligible immigrant pregnant women from different socio-economic backgrounds were routinely followed in the antenatal clinics (booked). Immigrant noneligible pregnant women who were not booked during pregnancy may present to the emergency department in established labor without any prior antenatal care (unbooked). The hospital's policy is to accept and admit them.

We included only women with singleton vertex pregnancies between 24-42 weeks of gestation who had one previous cesarean delivery and had undergone a trial of labor after cesarean section.

Women with previous two or more cesarean sections, antenatal intrauterine fetal demise, breech presentation, induction of labor, estimated fetal weight $>4 \mathrm{~kg}$, and classical or low vertical uterine incision were excluded.

The women were divided into four groups based on the number of trials of labor after cesarean section. Group 1 comprised those women with no previous history of the trial. Groups 2, 3, and 4 include those with the previous histories of one, two, three, or more than three trials of labor after cesarean section, respectively. Data extracted included maternal demographics, clinical characteristics, and labor and delivery outcomes (vaginal birth after cesarean success, uterine rupture, uterine dehiscence, need for blood transfusion (packed red blood cells), post-partum fever, APGAR scores, birth weight of the newborn, and neonatal intensive care unit admission). Uterine rupture was defined as a tear of the uterine muscle, and visceral peritoneum or a uterine muscle separation with extension to adjacent structures, and uterine dehiscence was defined as a disruption of the uterine muscle with intact serosa. The outcomes were compared according to the number of trials of labor after cesarean section.

Statistical analysis was conducted with SAS 9.4 (SAS Institute, Cary, NC). Mean, and standard deviation for continuous variables and frequency and proportions were calculated by the number of trials of labor after cesarean section. Trends of each variable with the trial of labor after cesarean section were tested. For a categorical variable, the Cochran-Armitage trend test was used if it had two levels, or the Mantel-Haenszel test was used if it had more than two levels. The normality test was performed for each of the continuous variables since all the variables are not normally distributed $(\mathrm{p}<0.05)$; the Jonckheere-Terpstra test was used to test trends. A two-tailed $\mathrm{p}$-value of $\mathrm{p}<0.05$ was considered statistically significant. 
Table I Demographic and Clinical Characteristics According to the Number of the Trial of Labor After Cesarean Section

\begin{tabular}{|c|c|c|c|c|c|c|}
\hline \multirow{2}{*}{\multicolumn{2}{|c|}{$\begin{array}{l}\text { Demographic and Clinical } \\
\text { Characteristics }\end{array}$}} & \multicolumn{4}{|c|}{ TOLAC, Number (Percentage) } & \multirow[t]{2}{*}{ P-value } \\
\hline & & Group I $(n=669)$ & Group $2(n=237)$ & Group $3(n=132)$ & Group $4(n=101)$ & \\
\hline \multicolumn{2}{|c|}{ Age (Mean $\pm S D)$} & $29.4 \pm 5.4$ & $30.6 \pm 5.5$ & $32.6 \pm 4.9$ & $35.4 \pm 4.8$ & $<0.000 \mathrm{I}^{\dagger}$ \\
\hline \multirow[t]{2}{*}{ Nationality } & Saudi & $585(87.4)$ & $193(8 I .4)$ & $93(70.4)$ & $23(22.7)$ & \multirow[t]{2}{*}{$<0.000 I^{*}$} \\
\hline & Non-Saudi & $84(12.5)$ & $44(18.6)$ & $39(29.6)$ & $78(77.2)$ & \\
\hline \multirow[t]{2}{*}{ Booking Status } & Booked & $519(78.0)$ & $175(74.2)$ & $99(75.6)$ & $78(78.0)$ & \multirow[t]{2}{*}{$0.6155^{*}$} \\
\hline & Non-booked & $146(2 \mid .9)$ & $61(25.9)$ & $32(24.4)$ & $22(22.0)$ & \\
\hline \multicolumn{2}{|c|}{ Gravidity (Mean \pm SD) } & $3.2 \pm 1.9$ & $4.1 \pm 1.7$ & $5.1 \pm I .4$ & $7.0 \pm 1.8$ & $<0.000 \mathrm{I}^{\dagger}$ \\
\hline \multicolumn{2}{|c|}{ Parity (Mean \pm SD) } & $1.8 \pm 1.5$ & $2.6 \pm 1.2$ & $3.4 \pm 0.9$ & $5.1 \pm 1.4$ & $<0.000 \mathrm{I}^{\dagger}$ \\
\hline \multicolumn{2}{|c|}{ BMI (Mean \pm SD) } & $30.8 \pm 6.5$ & $30.4 \pm 5.9$ & $30.9 \pm 5.7$ & $31.5 \pm 6.1$ & $0.7497^{\dagger}$ \\
\hline \multicolumn{2}{|c|}{ Hemoglobin before (Mean \pm SD) } & $11.1 \pm 1.3$ & $11.3 \pm 1.3$ & $11.3 \pm 1.3$ & $10.9 \pm 1.3$ & $0.6097^{\dagger}$ \\
\hline
\end{tabular}

Notes: Data are mean \pm standard deviation or $\mathrm{n}(\%)$. ${ }^{\mathrm{P}}$-values from Mantel-Haenszel test for trend or Cochran-Armitage Trend Test. ${ }^{\dagger} \mathrm{p}$-value from Jonckheere-Terpstra Test.

\section{Results}

During the study period, 1139 women met the study criteria and were included in the analysis. The number of women with zero, one, two, and three or more trials of labor after cesarean section were 669 (58.7\%), 237 (20.8\%), 132 $(11.6 \%)$, and $101(8.9 \%)$, respectively. The demographics and clinical characteristics were presented in Table 1. There were statistically significant trends between the four groups in age, nationality, gravidity, and parity but not in the booking status, BMI, and the hemoglobin level before a trial of labor after cesarean section. Mean age, gravidity, and parity significantly increased with the trial of labor after cesarean section. Simultaneously, the proportion of Saudi women decreased with the trial of labor after cesarean section from $87.4 \%$ after zero trial of labor after cesarean section to $22.8 \%$ after three trials of labor after cesarean section.

Significantly more women delivered vaginally $(p<0.001)$ with each attempted trial of labor after cesarean section, increasing from 488 women (72.9\%) with zero trial of labor after cesarean section to 221 women (93.3\%) with one trial of labor after cesarean section, 124 women $(93.9 \%)$ with two trials of labor after cesarean section, and 95 women (94.1\%) with three or more trials of labor after cesarean section (Table 2).

The uterine rupture occurred in one woman during the first trial of labor after cesarean section and in one woman

Table 2 Pregnancy Outcomes According to the Number of Trials of Labor After Cesarean Section

\begin{tabular}{|c|c|c|c|c|c|}
\hline \multirow[t]{2}{*}{ Pregnancy Outcomes } & \multicolumn{4}{|c|}{ TOLAC, Number (Percentage) } & \multirow[t]{2}{*}{ P-value } \\
\hline & Group I $(n=669)$ & Group $2(n=237)$ & Group $3(n=132)$ & Group $4(n=101)$ & \\
\hline VBAC Success & $488(72.9)$ & $221(93.2)$ & $124(93.9)$ & $95(94.0)$ & $<0.000$ I* \\
\hline Uterine Rupture & I $(0.1)$ & 0 & 0 & I (0.9) & $0.254 I^{*}$ \\
\hline Dehiscence & $4(0.6)$ & 0 & 0 & 0 & $0.1513^{*}$ \\
\hline Transfusion & $16(2.3)$ & $\mathrm{I}(0.4)$ & $\mathrm{I}(0.7)$ & $\mathrm{I}(0.9)$ & $0.0819 *$ \\
\hline Pyrexia & $10(1.4)$ & $\mathrm{I}(0.4)$ & $2(1.5)$ & 0 & $0.2372^{*}$ \\
\hline No of hospital stays (Mean $\pm S D$ ) & $2.4 \pm 1.7$ & $1.9 \pm 2.1$ & $1.8 \pm 1.3$ & $2.0 \pm 2.0$ & $<0.000 \mathrm{I}^{\dagger}$ \\
\hline Birth weight $(\mathrm{kg})$, (Mean \pm SD) & $3.1 \pm 0.5$ & $3.0 \pm 0.5$ & $3.2 \pm 0.5$ & $3.3 \pm 0.5$ & $0.0035^{\dagger}$ \\
\hline I-minute Apgar $<5$ & $20(2.9)$ & $\mathrm{I}(0.1)$ & $2(1.5)$ & I (0.9) & $0.0759 *$ \\
\hline 5-minute Apgar $<5$ & I $(0.1)$ & $237(100.0)$ & 0 & 0 & $0.4737^{*}$ \\
\hline Neonatal resuscitation & $4(0.6)$ & $\mathrm{I}(0.4)$ & 0 & I (0.9) & $0.9222^{*}$ \\
\hline ICU admission & $10(1.4)$ & 0 & I (0.8) & I (0.9) & $0.3168^{*}$ \\
\hline
\end{tabular}

Notes: Data are mean \pm standard deviation or $\mathrm{n}(\%)$. *P-values from Mantel-Haenszel test for trend or Cochran-Armitage Trend Test. ${ }^{\dagger} \mathrm{p}$-value from Jonckheere-Terpstra Test. 
with three or more trials of labor after cesarean section. Uterine dehiscence occurred only in four women $(0.6 \%)$ with no trial of labor after cesarean section. There were statistically significant differences in birth weights $(\mathrm{p}=$ $0.0035)$ and the duration of hospital stay ( $p<0.0001)$ between the groups, while other parameters, such as APGAR scores, neonatal resuscitation, and ICU admission, showed no significant differences between the groups (Table 2).

Mean birth weight increased with the number of trials of labor after cesarean section while the duration of hospital stays was longer when a trial of labor after the cesarean section was zero and shorter when a trial of labor after the cesarean section was more than zero $(2.49$ days vs $<2.0$ days).

\section{Discussion}

The safety of a trial of labor after one previous cesarean is well-documented. However, there are limited published reports on repeated trials of labor after the cesarean section as the national data is lacking and only a few old studies are available.

The main concern regarding repeat trials of labor after cesarean section had been the potential increase in maternal and neonatal morbidity. This concern was based on the theoretical risk that repeated trials of labor after cesarean section may produce additive strain on the uterine scar. The main finding of our study was that repeated trials of labor after cesarean section are safe for the mothers and the children. The number of successful vaginal deliveries increases with the number of attempted deliveries after the cesarean section from $72.9 \%$ with no previous trials of labor after cesarean section to $94 \%$ with three or more previous trials of labor after cesarean section.

Our study showed that a higher amount of attempted vaginal deliveries was not associated with an increased risk of uterine rupture. This result is consistent with the findings of Gyamfi et al ${ }^{10}$ and Mercer et al. ${ }^{11}$ The vaginal birth rate after cesarean was 94\% (318/336) in women with a history of one or more previous vaginal births after cesarean delivery as compared to $70.5 \%(620 / 880)$ in women without a history of vaginal birth after cesarean delivery $(\mathrm{P}<0.001)$. There was no significant difference in the uterine rupture rate in the previous vaginal birth after the cesarean delivery group compared with those without this history, $0.60 \%$ versus $1.93 \%$, respectively $(\mathrm{P}=0.093) .{ }^{10}$ Mercer et al, in 2008, published a secondary analysis of a 4-year observational study in 19 academic medical centers in the United States between 1999 and 2002. ${ }^{11}$ The study included 13,532 women with singleton pregnancies who had previously undergone at least one cesarean delivery by a low transverse uterine incision and who attempted a trial of labor after cesarean section. After a cesarean delivery, women with no prior vaginal birth had a $63.3 \%$ vaginal delivery rate; women with one prior vaginal birth after cesarean had a rate of $87.6 \%$, and those with two or more vaginal births after cesarean had a rate of $90.9 \%$. The rate of uterine rupture decreased after the first successful vaginal birth after cesarean and remained similarly low as the vaginal birth after cesarean number increased from two to four or more. After cesarean delivery, women with no previous vaginal birth had a uterine rupture risk of $0.87 \%$, while women with one previous vaginal birth after cesarean had a rupture risk of $0.45 \%$. Women with two or more vaginal births after cesarean had a uterine rupture risk of $0.43 \%$. Mercer et al's published study is the most extensive study demonstrating that the vaginal birth after cesarean success rate improved with repeat trials of labor after cesarean section. $^{11}$ Vaginal births after cesarean were associated with a lower risk of uterine rupture and perinatal complications.

The success rate of trial of labor after cesarean section is high ranging from $60-80 \%$ with low rates of complications. ${ }^{12}$ Serious complications like rupture uterus occur in $0.4-0.7 \%$. Uterine rupture is increased in the classical and the low vertical uterine incision, induction of labor with prostaglandins, and augmentation with oxytocin. Successful trial of labor after the cesarean section has several benefits over the elective cesarean section in the index and future pregnancies. However, these complications are highest when a trial of labor after cesarean section fails and the emergency cesarean section is performed. This, among other reasons, has led to a decrease in the trial of labor after cesarean section rate despite the existing recommendations of the major professional medical organizations supporting and encouraging women to have a trial of labor after cesarean section. Interestingly, there are significant variations in the trial of labor after cesarean section rates between countries. The rate of trial of labor after cesarean section in Japan is 5\%, 12-14\% in the United States and Australia, 29-36\% in Ireland, Italy, Germany, and $45-55 \%$ in Finland, Sweden, and the Netherlands. ${ }^{13-16}$ A recent study from a center in Israel reported a rate of $75.6 \% .{ }^{17}$ Cultural differences may provide some explanation for the varying trial of labor after cesarean section rates. ${ }^{18}$ 
In the present study, we have compared the success rate and the safety of repeat trials of labor after cesarean section in women with only one previous cesarean section, and we have excluded women with labor induction, whereas, other previous studies have included women with more than one previous cesarean section and labor induction. We found a statistically significant association between increasing maternal age and the number of prior trials of labor after cesarean section. Similar findings concerning maternal age were obtained by Mercer et al having a mean age of 28 years, 29.2 years, and 30.6 years for those with a history of no, one, and two or more vaginal births after cesarean section respectively $(p<0.001) .{ }^{11}$

In our study, the mode of delivery was significantly associated with the number of prior successful trials of labor after cesarean section. After a cesarean section, women who had a previous history of vaginal birth were more likely to repeat labor trials after cesarean section successfully. The rate of uterine rupture decreased from $0.15 \%$ to $0 \%$, with an increase in the number of vaginal births after cesarean, but after three or more vaginal births after cesarean, the uterine rupture tends to increase to $0.99 \%$.

Uterine dehiscence rate decreased from $0.6 \%$ for those with zero trial of labor after cesarean section to $0 \%$ for those with one, two, three, or more trials of labor after cesarean section. The small numbers of uterine rupture and dehiscence in our study preclude any meaningful comparisons. We found that the need for transfusion decreased during subsequent trials of labor after cesarean section, but this was not statistically significant. Mercer et al found a statistically significant association between the need for transfusion and the number of prior vaginal births after cesarean $(p=0.002) .{ }^{11}$ The need for blood transfusion decreases from 1.89 (no prior vaginal birth after cesarean) to 1.24 (one prior vaginal birth after cesarean) and 0.99 (two or more prior vaginal births after cesarean). In our study, there was no statistically significant increase between repeat trials of labor after cesarean section and neonatal morbidity.

There are some limitations to our study. Firstly, our study is of retrospective nature and depends solely on the medical records of the hospital. Secondly, we did not have data on women who were eligible for a trial of labor after cesarean section but who declined and elected to proceed with an elective repeat cesarean delivery.

\section{Conclusion}

Previously successful vaginal births after cesarean delivery are associated with improved maternal and neonatal outcomes, decrease in rupture rates and the hospital stay of the mother in a subsequent trial of labor after cesarean section. Hence, we recommend that women with previous vaginal birth after one cesarean section should be offered repeat trials of labor.

\section{Acknowledgments}

This project was funded by the Deanship of Scientific Research (DSR) at King Abdulaziz University, Jeddah, Saudi Arabia under Grant Number 5-012/430. The authors, therefore, acknowledge, with thanks, DSR technical and financial support.

\section{Disclosure}

The authors report no conflicts of interest in this work.

\section{References}

1. Betran AP, Ye J, Moller AB, Zhang J, Gülmezoglu AM, Torloni MR. The increasing trend in caesarean section rates: global, regional and national estimates: 1990-2014. PLoS One. 2016;11:e148343. doi:10.1371/journal.pone. 0148343

2. Kenny LC, Lavender T, McNamee R, O'Neill SM, Mills T, Khashan AS. Advanced maternal age and adverse pregnancy outcome: evidence from a large contemporary cohort. PLoS One. 2013;8:e56583. doi:10.1371/journal.pone.0056583

3. Crane JM, Murphy P, Burrage L, Hutchens D. Maternal and perinatal outcomes of extreme obesity in pregnancy. J Obstet Gynaecol Can. 2013;35(7):606-611. doi:10.1016/S1701-2163(15)30879-3

4. Biswas A, Su LL, Mattar C. Caesarean section for preterm birth, and breech presentation and twin pregnancies. Best Pract Res Clin Obstet Gynaecol. 2013;27:209-219. doi:10.1016/j.bpobgyn.2012.09.002

5. Chen I, Opiyo N, Tavender E, et al. Non-clinical interventions for reducing unnecessary caesarean section. Cochrane Database Syst Rev. 2018;9. Art. No.: CD005528. doi:10.1002/14651858. CD005528.pub3

6. American College of Obstetricians and Gynecologists. ACOG Practice Bulletin no. 205. Vaginal birth after previous cesarean delivery. Obstet Gynecol. 2019;133:e110-27. doi:10.1097/AOG.000 0000000003078

7. Society of Obstetricians and Gynecologists of Canada. Clinical Practice Guideline No. 155. Guidelines for vaginal birth after previous Caesarean birth. J Obstet Gynaecol Can. 2019;41:992-1011.

8. Royal College of Obstetricians and Gynecologists. Birth after previous cesarean birth Green-top Guideline No. 45; 2015. Available from: https://www.rcog.org.uk/en/guidelines-research-services/guide lines/gtg45/. Accessed April, 2020.

9. McMahon MJ, Luther ER, Bowes WA. Comparison of a trial of labor with an elective second cesarean section. $N$ Engl $J$ Med. 1996;335:689-695. doi:10.1056/NEJM199609053351001

10. Gyamfi C, Juhasz G, Gyamfi P, Stone JL. Increased success of trial of labor after previous vaginal birth after cesarean. Obstet Gynecol. 2004;104:715-719. doi:10.1097/01.AOG.0000139516.43748.1b

11. Mercer BM, Gilbert S, Landon MB, et al. Labor outcomes with increasing number of prior vaginal births after cesarean delivery. Obstet Gynecol. 2008;111:285-291. doi:10.1097/AOG.0b013e31816102b9

12. Landon MB, Grobman WA. Vaginal birth after cesarean delivery. In: Landon MB, Driscoll DA, Jauniaux ERM, Galan HL, Grobman WA, Berghella VM, editors. Gabbe's Obstetrics Essentials: Normal and Problem Pregnancies. Elsevier; 2018:145-150. 
13. Takeya A, Adachi E, Takahashi Y, et al. Trial of labor after cesarean delivery (TOLAC) in Japan: rates and complications. Arch Gynecol Obstet. 2020;301:995-1001. doi:10.1007/s00404-020-05492-8

14. Triebwasser JE, Kamdar NS, Langen ES, et al. Hospital contribution to variation in rates of vaginal birth after cesarean. $J$ Perinatol. 2019;39:904-910. doi:10.1038/s41372-019-0373-2

15. Australian Institute of Health and Welfare 2018. Australia's mothers and babies 2016-in brief. Perinatal statistics series no. 34. Cat. no. PER 97. Canberra: AIHW. Available from: https://www.aihw.gov.au/ getmedia/7a8ad47e-8817-46d3-9757-44fe975969c4/aihw-per-97.pdf. aspx?inline=true. Accessed June, 2020.
16. Nilsson C, Lalor J, Begley C, et al. Vaginal birth after cesarean: views of women from countries with low VBAC rates. Women Birth. 2017;30:481-490. doi:10.1016/j.wombi.2017.04.009

17. Lipschuetz M, Guedalia J, Rottenstreich A, et al. Prediction of vaginal birth after cesarean deliveries using machine learning. $\mathrm{Am}$ $J$ Obstet Gynecol. 2020;222:613.e1-16. doi:10.1016/j.ajog.20 19.12.267

18. Lundgren I, Morano S, Nilsson C, Sinclair M, Begley C. Cultural perspectives on vaginal birth after previous cesarean section in countries with high and low rates - A hermeneutic study. Women Birth. 2020;33:e339-e347. doi:10.1016/j.wombi.2019.07.300

\section{Publish your work in this journal}

The International Journal of Women's Health is an international, peerreviewed open-access journal publishing original research, reports, editorials, reviews and commentaries on all aspects of women's healthcare including gynecology, obstetrics, and breast cancer. The manuscript management system is completely online and includes a very quick and fair peer-review system, which is all easy to use. Visit http://www.dovepress.com/testimonials.php to read real quotes from published authors. 